\title{
A HIGH-FREQUENCY SCHOTTKY DETECTOR FOR USE IN THE TEVATRON*
}

\author{
D.A. Goldberg and G.R. Lambertson, \\ Lawrence Berkeley Laboratory, \\ Berikeley, CA 94720
}

\section{DISCLAIMER}

\begin{abstract}
This report was prepared as an sccount of work sponsored by an ugency of the United States Government. Neither the United States Government nor any agency thereof, nor any of their employees, makes any warranty, express or implied, or assumes any legal liability or responsibility for the accuracy, completeness, or usefulness of any information, apparatus, product, or proces diaclowed, or represents that its use would not infringe privately owned rights. Reference herein to any apecific commercial product, process, or service by trade name, trademart, manufacturer, or otherwise does not necesarily constitute or imply its endorsement, recommeadation, of favoring by the United States Government or any igency thereol. The views and opinions of authors expresed herein do not necessarily stnte or reflect those of the United States Gowerament or any agency thereof.
\end{abstract}

* Work supported by the Director, Office of Energy Research, Office of High Energy and Nuclear Physics, High Energy Physics Division, U.S.D.O.E., under Contract No. DE-AC03-76SF00098. 


\title{
A HIGH-FREQUENCY SCHOTTKY DETECTOR FOR USE IN THE TEVATRON*
}

\author{
D.A. Goldberg and G.R. Lambertson \\ Lawrence Berteley Laborasory, Berkeley CA 94720
}

\begin{abstract}
A vexing problem asociated with detection of Schouky signals from a bunched beam is the presence of the coherent signal, which can be 10 or more orders of magnitude greater than the Schottky siznal. To overcome this difficulty, we have conatructed a Schottky delector for the Tevatron collider is the form of a high-Q $(-5000)$ resonant cavity which operales at roughly $2 \mathrm{GHz}$, well above the frequency $a$ which the single-bunch frequency pectrum begins w roll off $(-200-300$ MHz for the Teveron). The devector is cepable of sensing independently the vertical and horizontal particle motions. The $2 \mathrm{GHz}$ Schottky signals are down-converted to frequencies below $100 \mathrm{kHz}$ to permit relatively rapid high-resolution andysis using = FFT spectrum enalyzer. The initial installation consists of a single cavity; a second detector will be built which employs a pair of phased cavities to permit discriminetion between p's and p's. Detnils of the design of both the cavity and the associaled electronics are presented. Spectrn oblained from the deteclor show clearly observable Schotthy betutron lines, free of coherent contaminants; also seen are the "common-mode" longitudinal sigmals due to the offet of the beam from the detector center. The latter signals indicate that at 2 GHz, the coherent single-bunch spectrum from the delector is reduced by $>80 \mathrm{~dB}$; therefore, in normal collider operation, the Schouthy betatron lines are essentially entirely free of coherent contaminants. Experimental data will be presented showing how the detector spectra can be used to measure such properties as transverse emittance and synchrotion frequency.
\end{abstract}

\section{INTRODUCTION}

With the use of a suitable detector, one can sense fluctuations in the instantaneous number and/or positions of particles in a cyclic accelerator. The signals from such a detector, the so-called Schotky signals, have as their principal virtue that they can furnish a variety of diagnostic information about a particle beam, on a continuous, real-time basis, without perturbing the beam.

The nature of Schottky signals is described in detail in Ref. 1. Summarized briefly, the froquency spectrum of these signals consists of a set of bands occurring at integer multiples of the particle revolution frequency, and a second set which is shifted in frequency from the first set due to the particles' betatron motion; if the beam is bunched, the synchrotron motion splits these t:ixds into a set of possibly overlapping satellite lines.

A vexing problem associated with detection of Schottiky signals from a bunched beam is the presence of a coherent signal, which can be 10 or more orders of magnitude greater than the Schottky signal. To overcome this difficulty, we have constructed a Schotky detector for the Tevatron collider, sensitive to transverse motion in both planes, in the form of a high-Q $(-5000)$ cavity whose resonant frequencies (made slightly different for the vertical and horizontal sensing modes) are roughly $2 \mathrm{GHz}$, well above the frequency at which the coherent single-bunch frequency spectrum begins to roll off $(-200-300 \mathrm{MHz}$ for the Tevatron), yet still below the cutoff frequency of the Tevatron beam pipe.

To achieve the frequency resolution necessary for measuring the widths of the central synchrotron satellite lines $(<3 \mathrm{~Hz})$, and still provide reasonable data acquisition times, it is necessary to employ a FFT spectrum analyzer. This in turm necessitates heterodyning the $2 \mathrm{GHz}$ cavity signal to something below $100 \mathrm{kHz}$,. To minimize spectral smearing due to phase noise from the beam, the heterodyning signal

\footnotetext{
"Work supported by the Director, Office of Energy Research, Office of High Energy and Nuclear Physics, High Energy Physics Division, U.S. D.O.E., under Contract No. DE-AC03-76SF00098.
} 
is referenced to an RF signal from the Tevatron; because of the difficulties of making such a receiver tunable, it was decided to tune the cavity to the (fixed) receiver frequency, rather than vice versa.

The initial detector consists of a single cavity of rectangular cross section, which responds equally to $p^{\prime} s$ and $p^{\prime} s$. Based on the success of this device, we are planning to build a second detector, which will employ a pair of phased cavities to permit discrimination between $p$ 's and $p$ 's. Our initial hope was that the detector be usable for both the fixed-urget and collider modes of the Tevatron; however, in fixed turget mode there appears to be a coherent signal from sources other than the bunch stricture per se, (presumably intra-bunch coherent oscillation) of sufficient magnitude as to preclude observation of Schottky signals.

\section{DETECTOR REQUIREMENTS}

The principal requirements for the Schottly detector are summarized in Table 1. In the main, they arise from the considerations discussed above, in conjunction with the Tevatron parameters given in Table 2.

Table 1 Design Goals

\section{SN}

fo (nominal cavity center frequency)

$\Delta f_{v, h}$ (vertical, horizonal frequency offsets)

Qu

sllowed frequency variation

sllowed tempenume varivion

\section{$>10 \mathrm{~dB}$}

$2044.5 \mathrm{MHz}$

\pm 2 MHz

10,000

$\pm 50 \mathrm{kHz} \quad$ (single cavity)

$\pm 15 \mathrm{kHz} \quad$ (duble cavity)

$\pm 1.0^{\circ} \mathrm{C} \quad$ (single cavity)

$\pm 25^{\circ} \mathrm{C} \quad$ (double cavity)

The above signal-to-noise ratio $(\mathrm{S} / \mathrm{N})$ is imposed on a machine operating to the specifications of Table 2; this makes it possible for the device to provide useful information for a machine operating at for example 1/10 of the stated intensity. Several factors governing the choice of operating frequency have already been discussed. In addition, to minimize the strength of the coherent signal in many-bunch operation, as well as to simplify the heterodyning scheme, we chose the cavity frequency to be a half-integer multiple of the If frequency; the multiple of 38.5 gives a frequency which is sufficiently below the beam tube cutoff frequency that the singledetector response is relatively unaffected by the beam tube ports, and that it will provide adequate isolation between two-cavities separated by no more than $50 \mathrm{~cm}$.

Tabie 2 Tevatron Operating Paraneiers (Collider Mode)

$f_{\text {tor }}$ (beam revolution frequency)

$\eta \quad$ (frequency/momentum dispersion factor)

Ap/p (fructional momenum spread)

$N$ (number of particles)

$\sigma_{\perp}$ (ms transverse beam size)
$47.71 \mathrm{kHz}$

.0028

$3 \times 10^{-4}$ (full width @ $900 \mathrm{GeV}$ )

$6 \times 10^{10}$ (per bunch)

$0.6 \mathrm{~mm}$ (radius) 
The dimensions of the rectangular cavity were chosen to displace the $\mathrm{TM}_{120}$ and $\mathrm{TM}_{210}$ frequencies by $\pm 2 \mathrm{MHz}$ from the above $f_{o}$. This spliting is low enough to permit reasonably narrow bandwidth in both the RF and 1st IF stages (see circuit discussion below), and yet high enough to avoid incidental coupling between the two modes; since the splitting frequency is also used as the frequency of the final IF stage, it should be high enough to permit signals from that stage to be transmitted over long distances without interference from the commercial AM band.

To translate the $S / \mathbb{N}$ requirement for the cavity into a $Q$-value we use the fact that the signal power (at frequency $f$ ) developed by a transverse pickup is given by

$$
P=\left\langle\left(I_{B} x\right)^{2}\right\rangle R_{\perp} T^{2}(\pi f / c)^{2}
$$

where $\left\langle\left(I_{B} x\right)\right\rangle$ is the Ims of the product of beam current and beam displacement, and $R_{\perp} T^{2}$ is the product of the transverse shunt impedance and transit-time factor. For a circular accelerator with particle revolution frequency $f_{\text {rev }}$ and a circulating beam of $N$ particles, the "source term" for the Schottky signal (per Schottky band) is given by

$$
\left\langle\left(I_{B} x\right)^{2}\right\rangle=N\left(e f_{\text {rev }}^{2}\right) \sigma_{\perp}^{2}
$$

Note that because Schottky signals result from fluctuations, this term is proportional to $N$ (for many accelerators, $N$ usually exceeds $10^{10}$ ), rather than $N^{2}$ as would be the case for coherent signals (this accounts for the ability of signals due to coherent motion to completely overwhelm the Schottky signals); for the same reason, the signal is proportional to the r.m.s. displacement of the individual particles (i.e. the beam size) rather than that of the beam as a whole, as would be the case for a coherent signal.

When excited in the $\mathrm{TM}_{210}$ (or $\mathrm{TM}_{120}$ ) mode, a closed rectangular cavity of length $\boldsymbol{\ell}$, an unloaded $Q$ of $Q_{u}$, and a matched output, has shunt impedance at resonance given by

$$
R_{\perp} T^{2}=\frac{64}{25 \pi^{2}} Z_{o} Q_{u} T^{2} \frac{\pi f \ell}{c}
$$

where $Z_{0}$ is the impedance of free space, and $T$ is the usual transit-time factor $\sin (\pi f \boldsymbol{L} / c) /(\pi f \boldsymbol{l} / c)$.

For the case of a cavity with beam ports at the end, Eq. 3 remains approximately correct as long as the port size remains small with respect to the wavelength. The product $T^{2}(\pi f \ell / c)$ has a broad maximum of $\approx 0.7$ at $\pi f \ell / c=1.17$, giving $R_{\perp} T^{2} / \mathrm{Q} \approx 34$ ohms. Using the Tevatron operating parameters given in Table 1 , and assuming a $3 \mathrm{~dB}$ noise figure for the electronics (amplifier noise plus cable attenuation), we find that an unloaded $Q$ of 10000 gives us a $S / N$ of $>13 \mathrm{~dB}$. Finally, a loaded $Q$ of 5000 gives a response which is $>8$ Schottky bands wide (FWHM) so that the detector response is essentially flat over the span of the central Schottky band.

The final requirements on tuning and temperature regulation are linked: The temperature stability is simply what is required to maintain the tuning tolerance. For the double cavity, the tolerance is based on the requirement that gains and phases be sufficiently matched to give $30 \mathrm{~dB}$ directional rejection; for the single cavity the tolerance is somewhat more arbitrary, and is based on keeping the response constant to $5 \%$. In either case, the required temperature tolerances exceed those of the available water systems at Fermilab, and we decided that the entire assembly be installed in a thermostatically controlled box to be maintained at $110^{\circ} \mathrm{F}$, roughly $10^{\circ}$ higher than the warmest anticipated ambient temperature in the Tevatron tunnel. 


\section{EXPERIMENTAL APPARATUS}

\subsection{Detector}

The single cavity detector is shown in Fig. 1; the interior of the device consists of a rectangular cavity with rounded corners. Attached to either end of the cavity are beam tubes which also serve to support the structure when it is installed in the Tevatron. The requirements of high $Q$, good vacuum properties, mechanical stability, and reliability and ease of fabrication led to a choice of 6061 aluminum for the cavity itself. Vacuum joints involving aluminum surfaces are made using a radially expanding metal (REM) seal, somewhat similar to a Mott seal; the REM seal also serves as an RF joint.

The tuning requirements for the cavity cannot be achieved by machining tolerances alone (the sensitivity to transverse cavity dimension is approximately $\mathbf{3 3 0}$ $\mathrm{kHz} / \mathrm{mil}$ ), and so a pair of micrometer-controlled tuning plungers, shown in the figure, must be employed. Each of the coupling antennas consists of an axial rod mounted on the end of an SMA fitting located roughly half way between the cavity center and its outer wall. Both the tuning plungers and the antennas, as well as a third antenna for injecting test signals, come mounted on con-flat flanges. To minimize the number of REM seals, the cover plate containing these feed-throughs was fabricated from a plate made of $1 / 2$ " Al bonded to 3/8" stainless steel. The former metal provided the required low resistivity interior surface; the latter permitted the use of con-flat seals for all feedthroughs.

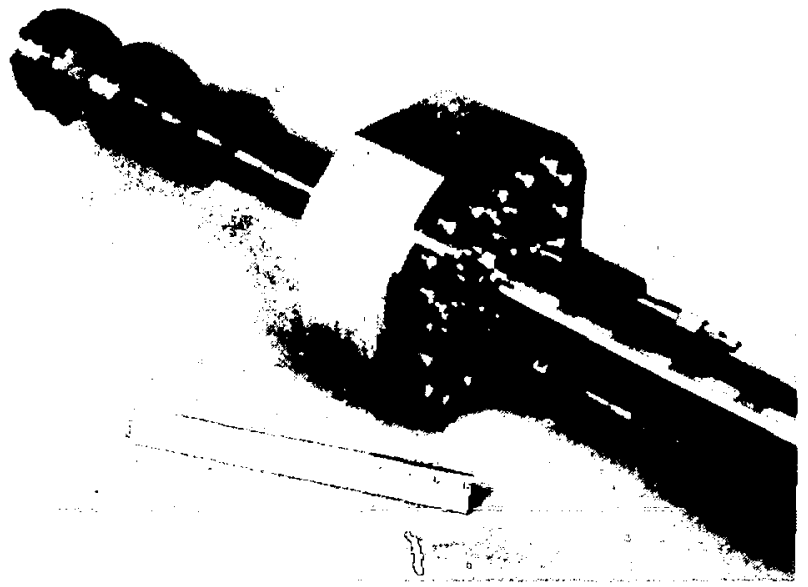

FIGURE 1 Assembled Schottky Detector

To tune and test the cavity, we first modified the antenna lengths so that they provided matched coupling to the external 50-ohm loads. The tuning plungers were then adjusted to give the proper "vertical" and "horizontal" frequencies (compensated 
for the system being at room temperature and not under vacuum). We then measured the unloaded Q-values and found them to be $Q_{v}=9500$ and $Q_{h}=9200$; the small difference is attributed to slight differences in the antennas and tuning probe positions. We then measured the detector response using the bead measurement technique described in the Ref. 2 , and obtained an $R T^{2} / \mathrm{Q}$ of $(29 \pm 1)$ ohms. The reduction from the calculated value of 34 is due principally to the reduction of the fields near the cavity ends due to the beam apertures; together with the slightly low $Q$ values, this results in a reduction of $S / N$ of just over $1 \mathrm{~dB}$ to a still acceptable value of $12 \mathrm{~dB}$.

\subsection{Electronics}

The aircuit for converting the $2 \mathrm{GHz}$ Schottky signal to a signal at $<100 \mathrm{kHz}$ is shown in Fig. 2; selected data on the receiver and the various signal levels and frequencies is given in Table 5 . The receiver's input if stage can switch-select either the vertical or horizontal signals from either $p$ 's or (after the double cavity is installed) $p$ 's. To minimize $\mathbf{S} / \mathrm{N}$ degradation, the switches are placed after the low-noise $(<1$ dB NF) Miteq input amplifiers. The only component preceding the amplifiers is a band-pass filter needed to block out the strong coherent longitudinal signal due to excitation of the cavity's $\mathrm{TM}_{110}$ mode, which would otherwise drive the input stage into saturation; the $S / N$ degradation resulting from these filters is only a few tenths of a dB.

Separate narrow-band filters for the vertical and horizontal signals in the first IF stage serve two purposes: They reduce noise power to the second mixer, thereby permitting additional gain in this stage; they also prevent the $3 \mathrm{~dB}$ degradation in $\mathbf{S} / \mathrm{N}$ which results from the folding of the noise spectrum onto the signal following mixing. The band pass filters in the second IF stage also serve this latter purpose. (It was not possible to find a sufficiently narrow band filter to employ this technique in the if stage as well.)

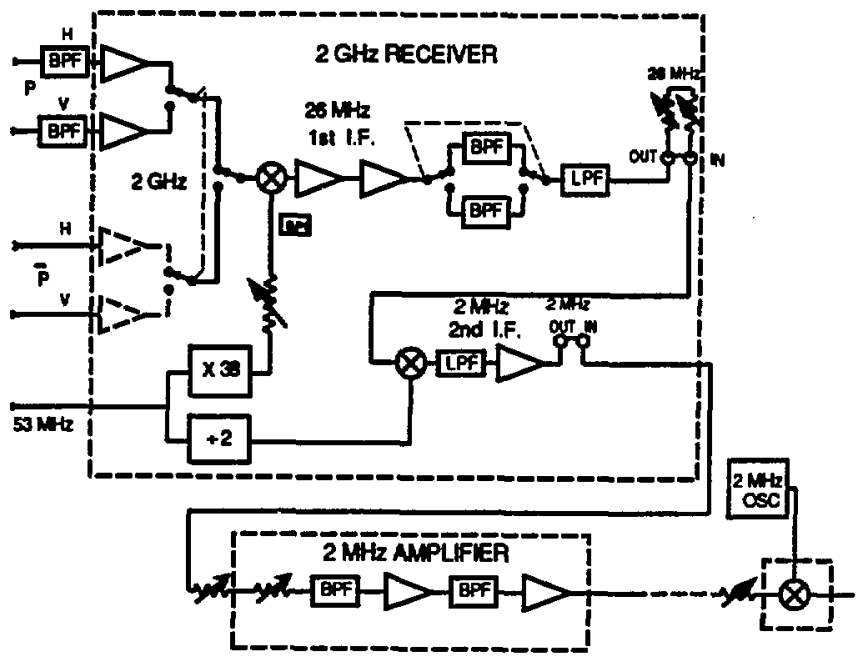

FGURE 2 Frequency Converter Block Diagram 
Input Signal Power (includes input line losses):

For $\mathrm{N}=10^{12}, \sigma_{\perp}=1 \mathrm{~mm}, 2800 \mathrm{~Hz}$ signal bundwidth:

$P=-116 \mathrm{dBm}$ per Schotiky betatron line

dPlä = -151 dBm/Hz (averaged over betaron line)

Input Noise Power Density $(T=295 \mathrm{~K})$ :

$$
\text { dPAf }=-173 \mathrm{dBm} / \mathrm{Hz} \text { (includes } 1 \mathrm{~dB} \text { NF for amplifier) }
$$

Receiver Conversion Gain: $86 \mathrm{~dB}$ (measured at output of $2 \mathrm{MHz}$ emplifier)

Receiver Openaing Frequencies:

Inpux if $2.04 \mathrm{GHz}$ (nominal) [Cavity frequencies: [ $f_{h}=2042.53, f_{v}=2046.53 \mathrm{MHz}$ ]

Iit IF 24.55 MHz, 28.55 MFz [f $f_{h}$ and $\left.f_{y} ; \Delta f-300 \mathrm{kHz}\right]$

2nd IF $2 \mathrm{MHz} ; \Delta \mathrm{f}=100 \mathrm{kHz}$

Buebend $0.100 \mathrm{kHz}$

Phase Noise $\ll 1 \mathrm{~Hz}$

As noted earlier, both the vertical and horizontal signals get down-converted to the same $2 \mathrm{MHz}$ final IF. The final conversion stage is located on a separate chassis so that the 2 MHz signal can be used for transmitting from the detector location to the main control room, roughly $1 \mathrm{~km}$ away. The variable frequency oscillator in the firial conversion stage permits selecting different Schottky bands, and positioning of the desired band within the $100 \mathrm{kHz}$ frequency window of the spectrum analyzer.

To resolve the central synchrotron satellite lines, we need a frequency resolution small compared to the $38 \mathrm{~Hz}$ synchrotron frequency; to be able to measure the linewidths requires even better resolution. The principal limit to such resolution is phase noise introduced by either the beam or the measuring electronics. To minimize the effects of the former we have used a frequency conversion scheme referenced to the RF system; measurements on the beam (see below) indicated the system phase noise to be well below; $1 \mathrm{~Hz}$ FWHM.

Finally, a word may be in order on the number of attenuators present. The original receiver design was based on the input power levels shown in Table 5. Initial experiments showed significantly higher input levels due to the presence of coherent longitudinal signals (see Sect. 4), and it was necessary to reduce the gain at the later stages to avoid saturation of the electronics.

\subsection{Temperature control}

To achieve the required temperature stability the detector assembly is enclosed in an insulated box having 2 " styrofoam/plywood walls. The box temperature is maintained at $110^{\circ} \mathrm{F}$ by means of a thermostatically controlled heater (in the form of a long-life $150 \mathrm{~W}$ light bulb) and a muffin fan to ensure uniform air temperature. The control unit is an Omeg2 Model CN-2002-P2 controller, which has the capability of a programmably variable output to the heater. The temperature sensing element is a platinum RTD mounted on the detector. Initial tests indicated that the system can maintain the cavity temperature $10< \pm 0.2^{\circ} \mathrm{C}$.

\section{EXPERIMENTAL RESULTS}

Data were acquired during two separace Tevatron col'jder runs. The first of these was at $273 \mathrm{GeV}$ which utilized the "fixed-target" optics, for which the vertical 
and horizontal beta functions (beam sizes) at the detector location were approximately equal. The second run was at $900 \mathrm{GeV}$, and utilized the "low- $\beta$ " optics, for which $\beta_{\mathrm{v}}$ is $=8.5 \beta_{\mathrm{h}}$. A typical spectrum, obtained during the $273 \mathrm{GeV}$ run, is shown in Figure 3, and illustrates most of the important features of the data (as explained in the Appendix, we "used" only the upper portion of the spectrum).

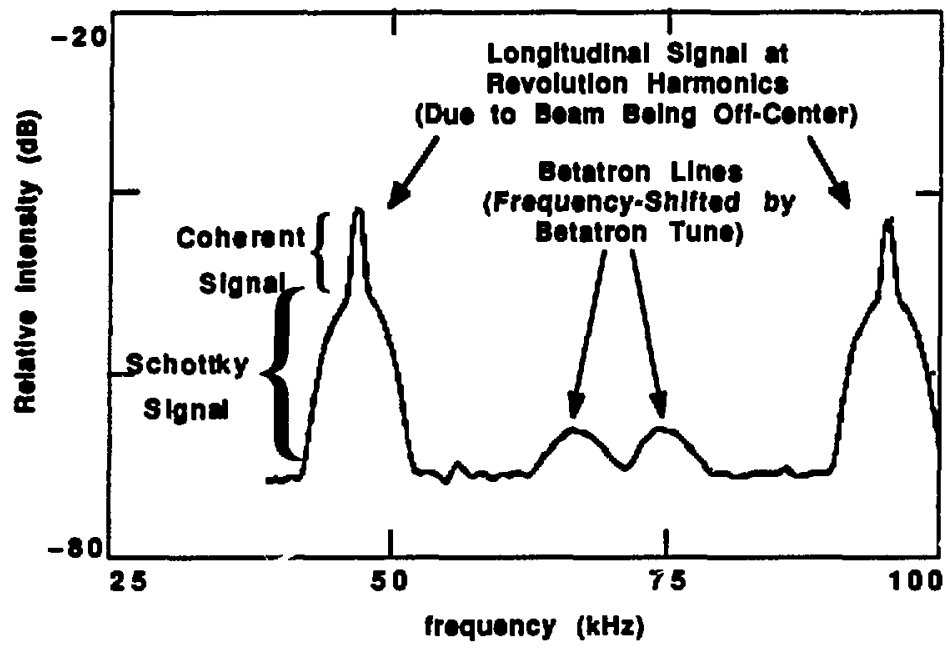

FIGURE 3 Spectrum of Vertical Output Signal from Schottly detector. E=273 GeV

The two large peaks at roughly 50 and $100 \mathrm{kHz}$ are the longitudinal signals, variously known as revolution lines or common-mode lines. Their presence is due to the fact that the beam is not centered in the detector, in the spectrum coming directly from the detector (i.e., prior to heterodyning) they occur at integer multiples of the 47.7 kHz revolution frequency; both of the peaks in Fig. 3 are at frequencies corresponding to "weak" coherent lines (see Appendix). We see that these are compound peaks-a narrow, intense peak atop a broad, weaker one. The strong, narrow peak is the residual coherent signal at $2 \mathrm{GHz}$; the broad peak is the longitudinal Schottky signal.

The two peaks appearing between the two revolution lines are the betatron signals. The line widths are comparable to those of the bruad peaks seen in the revolution lines, and there is no evidence of the narrow peak, i.e. they appear to be Schottky signals completely uncontaminated by any coherent signal!

The breadth of the Schottky line reflects the momentum variations of the individual particles within the beam, whereas that of the coherent line simply refleets the variation of the centroid of the beam, which is considerably less. The observed widths of the Schottly peaks are consistent with the expected momentum spreads of the beam. The identification of the broad and narrow peaks was inadvertently confirmed when the If anode supply failed during one of the beam stores; the resulting debunching of the beam caused the coherent (namow) peak to disappear immediately, where as the the Schottky signal was (initially) unaffected.

To assess the effectiveness of operating at high frequency to reduce the coherent signal, we make use of the expressions for the total power in the coherent and incoherent (Schottky) common mode lines. 


$$
P_{c} \propto\left\langle\gamma_{c}^{2}\right\rangle \cdot\left(x_{d}^{2}\right) \quad P_{S} \propto\left\langle\left(r_{S}\right\rangle\right) \cdot\left\langle x_{d}^{2}\right\rangle
$$

Since both signals are proportional to the mean-square beam displacement $\left\langle x^{2} d\right\rangle$, the ratio of the mean-square Schottky and coherent currents is simply the ratio of the measured powers, i.e. of the areas under the respective peaks. As discussed in Sect. 2 , at low frequencies this ratio is equal to $N$, the number of particles; hence dividing the above power ratio by $N$ gives the effective coherent signal suppression. Over a number of runs at both 273 and $900 \mathrm{GeV}$ at intensities of $25 \times 10^{11}$, we almost invariably found the strongest of the coherent peaks to be less than $35 \mathrm{~dB}$ greater than the Schortky peak, * meaning that at $2 \mathrm{GHz}$, the coherent signal is suppressed by more than $80 \mathrm{~dB}$. (We also observed an unexpected [and as yet unexplained] result: The observed width of the coherent lines decreased by roughly a factor of 2 over the first few hours of a beam store [the width of the Schottky lines remained essentially unchanged during the sume period], accompanied by the appearance and disappear- nee of gross structure in the line shape; there did not appear to be any correlated change in the amplitude of either the coherent or the Schottky peaks.)

We can now estimate the degree to which the Schottky betatron lines are free of coherent contaminants. Expressions for the total power in the coherent and Schottky betatron peaks are given by

$$
\left.P_{c}^{b} \propto\left\langle P_{c}^{2}\right\rangle \cdot\left\langle x_{B C}^{2}\right\rangle \quad P\right\} \propto\left\langle\langle z\} \cdot\left\langle x_{B i}^{2}\right\rangle\right.
$$

where $\left\langle x^{2} \beta c\right\rangle$ and $\left\langle x^{2} \beta\right\rangle$ are the mean-square coherent and incoherent betatron amplitudes, respectively. Hence in addition to the ratio of mean-square coherent to Schottky currents, the relative powers depend on the ratio $\left\langle x^{2} \beta_{c}\right\rangle /\left\langle x^{2} \beta_{i}\right\rangle$. We know from experiments done with the Tevatron tune detector ${ }^{3}$ that $\left\langle x^{2} \beta c\right\rangle \mid\left\langle x^{2} \beta_{i}\right\rangle$ $510^{-8}$, so that this additional suppression of $80 \mathrm{~dB}$ means that the coherent contaminant in the betatron lines is some $45 \mathrm{~dB}$ below the Schottky signal (even for the case where the contaminant is produced by a strong coherent betatron line), consistent with the apparent total absence of a coherent betatron peak.

The longitudinal lines in Fig. 3 are not in detail smooth curves but consist of a set of so-called synchrotron sidebands or satellites which result from the modulation of the particles' revolution frequency as a result of synchrotron oscillation. A greatly expanded view of the center of a revolution line (from the $900 \mathrm{GeV}$ data) is shown in Fig. 4, which shows the central line (equivalent to the "carrier frequency") as well as the first two satellites on either side. The separation of the satellites is equal to the frequency at which the revolution frequency is modulated, i.e. the synchrotron frequency; from the data in Fig. 4 we determine that to be $37.6 \pm 0.2 \mathrm{~Hz}$.

As we noter earlier, the ability to obtain resolution of better than $1 \mathrm{~Hz}$ on signals which are originally at $2 \mathrm{GHz}$ results from using the signal from the Tevatron if system as the source of the heterodyning frequency; the sharpness of the peaks also indicates that the receiver has very low phase noise. (The central satellite, theoretically infinitely narrow, has 2 width consistent with instrument resolution when viewed at resolution bandwidth as low as .05 Hz!) Hence one is able to observe the considerable fine itructure seen in Fig. 2. The fact that the structure is mirror-symmetric about the central satellite suggests that it is actually signal on the beam, and not either noise or an artifact of the heterodyning process. While the source(s) of the structure have

\footnotetext{
As discussed in the appendix, the relative strengths of the various coherent peaks in a given spectrum can very considerably, depending on the uniformity of the bunch populations. For the case shown in Fig. 3, the relative areas under the coherent and Schouky peaks differ by linte more then a factor of 2; however the ratio of the strong coherent line (not shown) wo the weak ones was nearly $30 \mathrm{~dB}$.
} 
not been identified, it is consistent with a group of particles within an annulus in the longitudinal phase space of the beam quite near to the outer edge of the rf bucket (not necessarily the same bucket as that containing the main portion of the beam); several individuals at Fermilab have independently suggested that such an annulus could be populated at the time the individual beam bunches are coalesced in the Main Ring.

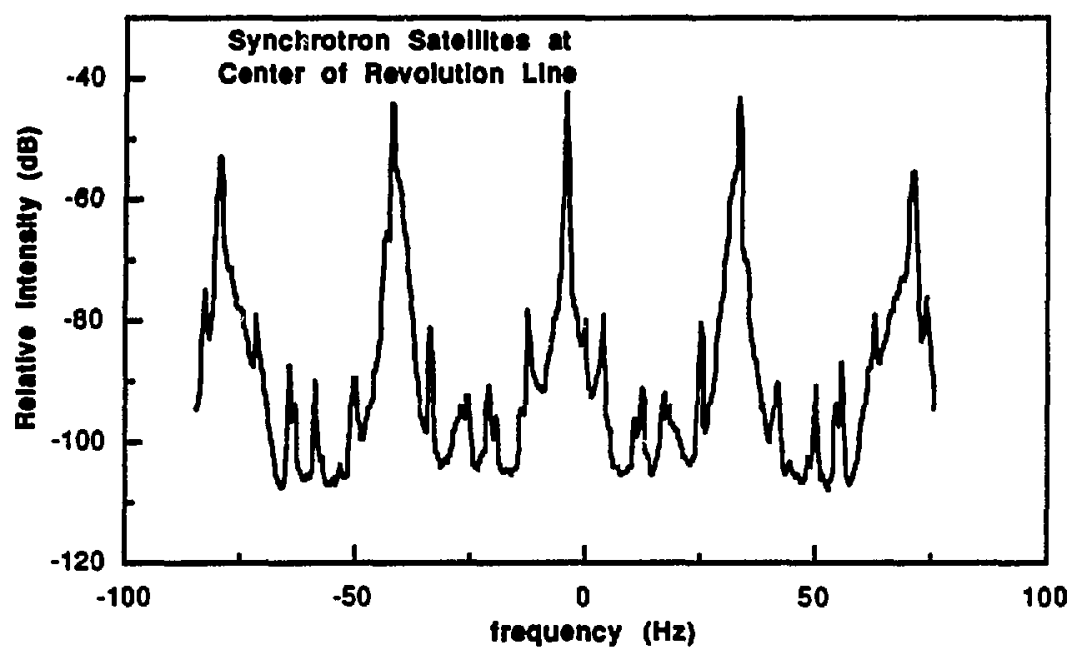

FIGURE 4 Central synchrotron savellies observed during operation at $=900 \mathrm{GeV}$.

One also expects to see satellite structure on the betatron peaks. When on'y protons are present we do indeed see them, with the same frequency spacing at which they are observed in the longitudinal spectrum. The lines (including the central peak) are much broader, about $11 \mathrm{~Hz}$ FWHM. The most likely cause of this is the variation of the tune with betatron amplitude; the present technique appears to be a particularly convenient way of measuring this quantity. When $p$ 's are introduced along with the protons, the satellite structure disappears, presumably smeared out due to the beambeam tune shift which, in addition to broadening the peaks, shifts those for p's and p's by different amounts. Were one to detect signals from p's and p's separately, it is possible that satellite structure could be recovered.

The final item for discussion is the betatron spectrum itself. The total power in the individual betatron lines, conveniently measured by viewing them with broad resolution, should provide a measure of the beam emittance. To see if this could be usefully done, we looked at the $S / N$ and found it to be within $1 \mathrm{~dB}$ of the calculated value (based on the measured emittance and beam current). We should note that due to the asymmetry of the beta functions in the "low- $\beta$ " mode, the S/N observed in the vertical spectrum is enhanced by roughly $3 \mathrm{~dB}$ over what it would be if one were using the more symmetric fixed-target optics (the obverse of that result is that the $S / N$ for horizontal betatron line is degraded in this mode, having a S/N value of roughly 8 $\mathrm{dB}$ lower than that of the vertical signal for the same operating conditions). However, we are advised that a new set of "low- $\beta$ " optics has been developed for the next collider run which has more nearly balanced beta functions, and should yield comfortable S $\mathbb{N}$ ratios for signals from both planes. 


\section{ACKNOWLEDGEMENTS}

We have benefitted greatly from the advice and counsel of Ferd Voelker, who did several feasibility studies on the initial idea of a resonant cavity detector. Design and construction of the cavity was done with the invaluable assistance of Tom Henderson and John Meneghetti. Similar assistance in the design and construction of the electronic circuits and the temperature control apparatus was provided by Walter Barry and Jim Wise. We are grateful for the encouragement and counsel of numerous Fermilab personnel throughout this project, most notably to Jim Crisp for his continual cooperation in his role as our Fermilab liaison, and to Gerry Jackson for his help in acquiring and digitally recording the data from the $900 \mathrm{GeV}$ run. In what is no doubt 2 departure from the usual procedure, we feel we must acknowledge the HewlettPackard Corporation for developing the microwave instrumentation without the aid of which this entire undertaking would have been impossible. Finally, we would like to acknowledge Jim Hinkson for numerous helpful suggestions on how to translate the jargon of beam physicists into a form intelligible to instrumentation engineers.

\section{APPENDLX}

\section{A NOTE ON THE PERIODICITY OF THE SCHOTTKY SPECTRA}

When the Tevatron colider is operated with three beam bunches (of $p$ 's and/or $\overrightarrow{\mathrm{p}} \mathrm{s}$ ), one expects strong coherert lines at intervals of $3 f_{\text {revi }}$ because of the nonuniform population of the individual bunches, weaker signals are also expected at the intermediate multiples of $f_{\text {rev. }}$ With six bunches, the same situation obtains, because the operating rf harmonic (1113) is not an integer multiple of 6 . The cavity is narmally tuned so that its peak responses (in both planes) lie midway between two of the $3 f_{\text {rey }}$ lines; over that $=150 \mathrm{kHz}$ span the cavity's response is flat to $- \pm 0.3 \mathrm{~dB}$.

Since the FFT analyzer can span $100 \mathrm{kHz}$, and the Schottky band spacing is only $47.7 \mathrm{kHz}$, the observed spectrum normally includes three revolution lines. However, due to an artifact of the final heterodyning stage, the lower half of the spectrum is generally distorted; consequently we analyzed only those data lying between (and including) the upper two revolution lines in any of the spectra. In the $273 \mathrm{GeV}$ data, the final frequency conversion was normally done so as to riake these both weak lines; for the $900 \mathrm{GeV}$ runs the lower of the two was usually a strong line.

\section{REFERENCES}

1. S. Chatopadhyay, "Some Fundamental Aspects of Fluctuations and Coherence in ChargedParticle Beans in Stornge Rings," CERN Report 84-11 (unpublished)

2. W. Barry and G.R. Lambertson, "Perturbation Method for the Measuremeris of Longitudinal and Transverse Betm Impedonce," et al, in Proc. 1987 IEEE Particle Accelerator Conf., p.1602.

3. G.P. Jackson, private communication. 